\title{
PREDUCTAL OXYGEN SATURATION TRENDS IMMEDIATELY AFTER BIRTH AND FEASIBILITY OF IMPLEMENTATION OF NRP 2010
}

\author{
A.B. Mahindre, A. Bhatnagar \\ Paediatrics \& Neonatology, Fortis Escort Hospital Faridabad Newdelhi, Faridabad, India
}

Background: The concept of normoxia is still not well defined. We definitely want a trend toward using "appropriate" oxygen concentrations for neonatal resuscitation. The goal is to keep the baby's oxygen saturation in the targeted levels. But is this feasible during initial phase of resuscitation?

Objective: To define the preductal oxygen saturations $\left(\mathrm{SpO}_{2}\right)$ trends and to find feasibility of maintaining targeted SPO2 immediately after birth.

Methods: All newborns delivered by NVD \&LSCS were included. $\mathrm{SpO}_{2}$ was measured with sensor placed on the right hand. Preductal oximetry data was downloaded and analyzed .Based on this data the percentile curves were drawn for normal vaginal and caesarian births.

Results: Data from 118 infants were analyzed. 70 newborns were caesarean babies \& 48 were normal vaginal deliveries.
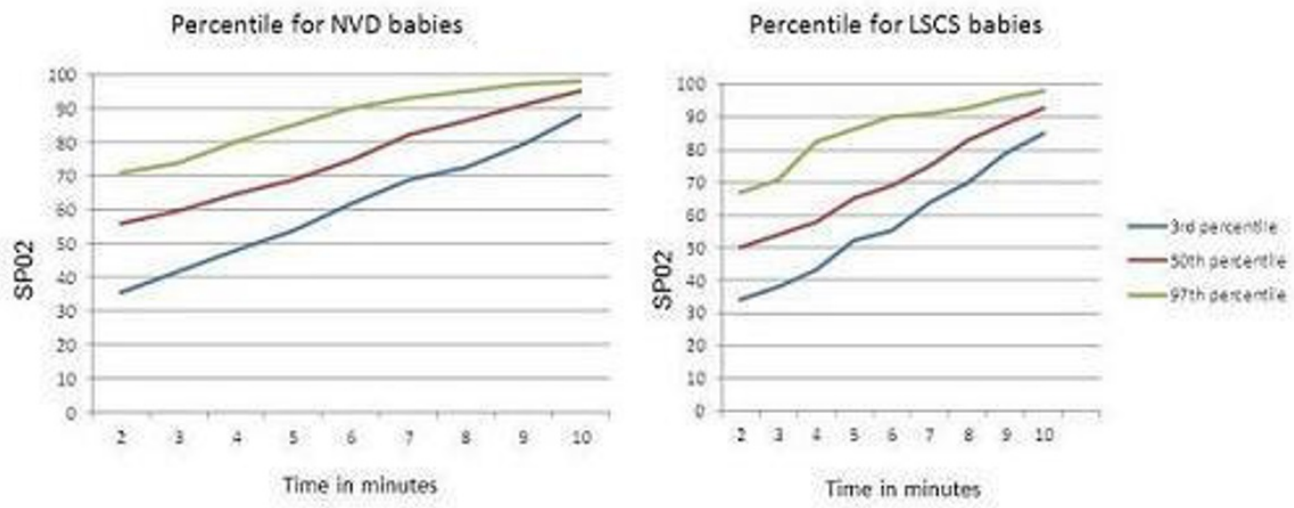

[percentile curves for NVD \& LSCS babies]

Potential limitation: Total study population 215,Areas of data dropout $14.8 \%$ Areas of rapid changes in saturation $12.09 \%$ Sensor misplacement $13.48 \%$ Need for supplemental O2 6.5\% Remaining sample size 118 i.e. $54.88 \%$.

Conclusion: Our results suggest that early oximetry readings, particularly in the first 60 seconds, must be interpreted with caution. Pulse oximetry could potentially play a role in adjusting the oxygen concentration beyond 2 minutes of life. The simplicity \& feasibility of this tool may be deceptive and there is still no substitute for clinical acumen. 